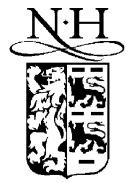

ELSEVIER

\title{
On the stability of delayed feedback controllers
}

\author{
Ömer Morgül \\ Bilkent University, Department of Electrical and Electronics Engineering, 06533 Bilkent, Ankara, Turkey \\ Received 27 January 2003; accepted 30 May 2003 \\ Communicated by A.P. Fordy
}

\begin{abstract}
We consider the stability of delayed feedback control (DFC) scheme for one-dimensional discrete time systems. We first construct a map whose fixed points correspond to the periodic orbits of the uncontrolled system. Then the stability of the DFC is analyzed as the stability of the corresponding equilibrium point of the constructed map. For each periodic orbit, we construct a characteristic polynomial whose Schur stability corresponds to the stability of DFC. By using Schur-Cohn criterion, we can find bounds on the gain of DFC to ensure stability.
\end{abstract}

(C) 2003 Elsevier B.V. All rights reserved.

PACS: $05.45 . \mathrm{Gg}$

Keywords: Chaotic systems; Chaos control; Delayed feedback; Pyragas controller

\section{Introduction}

Since the seminal work of [1], the possibility of controlling chaotic systems has received a great deal of attention among scientists from various disciplines including the physicists. In chaotic systems usually many unstable periodic orbits are embedded in their chaotic attractors, and as shown in [1], by using small external feedback input, some of these orbits may be stabilized. Therefore, by applying small external forces, it may be possible to obtain some regular behaviour in such systems. Following [1], various chaos control techniques have been proposed, [2,3].

E-mail address: morgul@ee.bilkent.edu.tr (Ö. Morgül).
Among these, the delayed feedback control (DFC) scheme first proposed in [4] and is also known as Pyragas scheme, has gained considerable attention due to its various attractive features. In this technique the required control input is basically the difference between the current and one period delayed states, multiplied by a gain. Hence if the system is already in the periodic orbit, this term vanishes. Also if the trajectories asymptotically approach to the periodic orbit, this term becomes smaller.

DFC has been successfully applied to many systems, including the stabilization of coherent modes of laser [5,6]; cardiac systems, [7,8], controlling friction, [9]; chaotic electronic oscillators, [10,11]; chemical systems, [12]. To overcome the limitations of DFC, several modifications have been proposed, [13-17]. 
Despite its simplicity, a detailed stability analysis of DFC is very difficult, $[16,18]$. For some recent stability results related to DFC, see [16-21].

In this Letter, we consider the delayed feedback control (DFC) scheme for one-dimensional discrete time systems. To analyze the stability, we construct a map whose fixed points correspond to the periodic orbits of the system to be controlled. Then the stability of the DFC is equivalent to the stability of the corresponding equilibrium point of the constructed map. For each periodic orbit, we construct a characteristic polynomial of a related Jacobian matrix. The Schur stability of this polynomial could be used to analyze the stability of DFC. By using Schur-Cohn criterion, we can find bounds on the gain of DFC to ensure stability.

This Letter is organized as follows. In Section 2 we present the basic form of DFC and some notation which will be used in this Letter. In the third section we will give our basic stability results. In the following section we will present some applications as well as some simulation results. Finally we will give some concluding remarks.

\section{Delayed feedback control}

Let us consider the following one-dimensional discrete-time system

$x(k+1)=f(x(k))+u(k)$,

where $k=0,1, \ldots$ is the discrete time index, $f: \mathbf{R} \rightarrow$ $\mathbf{R}$ is an appropriate function, which is assumed to be differentiable wherever required, and $u \in \mathbf{R}$ is the control input. We assume that in the uncontrolled case (i.e., when $u \equiv 0$ ) the system given by (1) possesses a $T$ periodic orbit characterized by the set $\Sigma_{T}=$ $\left\{x_{0}^{*}, x_{1}^{*}, \ldots, x_{T-1}^{*}\right\}$, i.e., for $x(0)=x_{0}^{*}$, the iterates of (1) with $u \equiv 0$ yields $x(1)=x_{1}^{*}, \ldots, x(T-1)=x_{T-1}^{*}$, $x(k)=x(k-T)$ for $k \geqslant T$. Let us call this orbit as an uncontrolled periodic orbit (UCPO) for future reference.

Let $\mathcal{S} \subset \mathbf{R}$ be a set, and $y \in \mathbf{R}$. We define the distance $d(y, \mathcal{S})$ between $y$ and $\mathcal{S}$ as

$d(y, \mathcal{S})=\inf _{z \in \mathcal{S}}|y-z|$.

We say that $\Sigma_{T}$ is asymptotically stable if for some $\epsilon>0$, for any $y \in \mathbf{R}$ satisfying $d\left(y, \Sigma_{T}\right)<\epsilon$, the iterates of (1) with $x(0)=y$ yields $\lim _{k \rightarrow \infty} d(x(k)$, $\left.\Sigma_{T}\right)=0$. We say that $\Sigma_{T}$ is exponentially stable if this decay is exponential, i.e., the following holds for some $M>0$ and $\rho \in(0,1)$

$d\left(x(k), \Sigma_{T}\right) \leqslant M \rho^{k} d\left(y, \Sigma_{T}\right)$.

In DFC, the following simple feedback control input is used to stabilize $\Sigma_{T}$ :

$u(k)=K(x(k)-x(k-T))$,

where $K \in \mathbf{R}$ is a constant gain to be determined. Note that if $x(0) \in \Sigma_{T}$, then $x(k) \in \Sigma_{T}$ and $u(k) \equiv 0$. Moreover, if $\Sigma_{T}$ is asymptotically stabilized, then $u(k) \rightarrow 0$ as $k \rightarrow \infty$. In the sequel we will derive some conditions and bounds on $K$ for the stabilization of periodic orbits.

\section{Stability analysis}

To motivate our analysis, consider the case $T=1$. In this case we have $\Sigma_{1}=\left\{x_{0}^{*}\right\}$ where $x_{0}^{*}=f\left(x_{0}^{*}\right)$, i.e., period 1 orbits are the same as fixed points of $f$. By defining $x_{1}(k)=x(k-1), x_{2}(k)=x(k)$, we can rewrite (1) and (4) as

$x_{1}(k+1)=x_{2}(k)$,

$x_{2}(k+1)=f\left(x_{2}(k)\right)+K\left(x_{2}(k)-x_{1}(k)\right)$.

Let us define $\hat{x}=\left(x_{1} x_{2}\right)^{T} \in \mathbf{R}^{2}$, where superscript $T$ denotes transpose, and define $F: \mathbf{R}^{2} \rightarrow \mathbf{R}^{2}$ as $F(\hat{x})=$ $\left(x_{2} Y_{2}\right)^{T}$, where $Y_{2}=f\left(x_{2}\right)+K\left(x_{2}-x_{1}\right)$. For $\hat{x}^{*}=$ $\left(x_{1}^{*} x_{2}^{*}\right)^{T}, F\left(\hat{x}^{*}\right)=\hat{x}^{*}$ holds if and only if $x_{1}^{*}=x_{2}^{*}=$ $f\left(x_{2}^{*}\right)$. Hence any fixed point of $F$ corresponds to an UCPO $\Sigma_{1}$ of (1), and vice versa. Hence asymptotic stability of $\Sigma_{1}$ for (1) and (4) can be analyzed by studying the stability of the corresponding fixed point of $F$ for (5). To analyze the latter, let $\Sigma_{1}=\left\{x_{0}^{*}\right\}$ and set $a_{1}=D f\left(x_{0}^{*}\right)$, and $J=\left.\frac{\partial F}{\partial x}\right|_{\Sigma_{1}}$, where $D$ stands for the derivative and $J$ is the Jacobian of $F$ evaluated at the equilibrium point. Clearly the components of $J$ are given as $J(1,1)=0, J(1,2)=1, J(2,1)=$ $-K, J(2,2)=a_{1}+K$. The characteristic polynomial $p_{1}(\lambda)$ of $J$ can easily be found as

$p_{1}(\lambda)=\operatorname{det}(\lambda I-J)=\lambda^{2}-\left(a_{1}+K\right) \lambda+K$.

We say that a polynomial is Schur stable if all of its eigenvalues are inside the unit disc of the complex 
plane, i.e., have magnitude less than unity. Hence, the asymptotic stability of the fixed point of $F$ for (5), hence the asymptotic stability of $\Sigma_{1}$ for (1) and (4) could be analyzed by studying the Schur stability of $p_{1}(\lambda)$ given by (6). Moreover note that the exponential stability of the fixed points of $F$ is equivalent to Schur stability of $p_{1}(\lambda),[22]$. Hence we can state the following facts:

1: The UCPO $\Sigma_{1}$ is exponentially stable for (1) and (4) if and only if $p_{1}(\lambda)$ given by (6) is Schur stable. This condition is only sufficient for asymptotic stability of $\Sigma_{1}$.

2: If $p_{1}(\lambda)$ has an unstable root, i.e., outside the unit disc, then $\Sigma_{1}$ cannot be asymptotically stable for (1) and (4).

Remark 1. We note that Schur stability of a polynomial can be determined by checking some inequalities in terms of its coefficients; this is known as the Jury test, see [23]. We will apply this test to (6) later.

To motivate our approach further, let us consider the case $T=2$. Let the period 2 UCPO of (1) be given as $\Sigma_{2}=\left\{x_{0}^{*}, x_{1}^{*}\right\}$ and define $a_{1}=D f\left(x_{0}^{*}\right), a_{2}=$ $D f\left(x_{1}^{*}\right)$. By defining $x_{1}(k)=x(k-2), x_{2}(k)=x(k-$ $1), x_{3}(k)=x(k)$, we can rewrite (1) and (4) as

$x_{1}(k+1)=x_{2}(k), \quad x_{2}(k+1)=x_{3}(k)$,

$x_{3}(k+1)=f\left(x_{3}(k)\right)+K\left(x_{3}(k)-x_{1}(k)\right)$.

For $\hat{x}=\left(x_{1} x_{2} x_{3}\right)^{T} \in \mathbf{R}^{3}$, let us define $G: \mathbf{R}^{3} \rightarrow \mathbf{R}^{3}$ as $G(\hat{x})=\left(x_{2} Y_{1} Y_{2}\right)^{T}$ where $Y_{1}=x_{3}, Y_{2}=f\left(Y_{1}\right)+$ $K\left(Y_{1}-x_{1}\right)$. Note that the fixed points of $G$ do not correspond to the UCPOs of (1), but the fixed points of $F=G^{2}$ does. To see this, note that $F=$ $\left(Y_{1} Y_{2} Y_{3}\right)^{T}$ where $Y_{3}=f\left(Y_{2}\right)+K\left(Y_{2}-x_{2}\right)$. For $\hat{x}^{*}=\left(x_{1}^{*} x_{2}^{*} x_{3}^{*}\right)^{T}$, the fixed points of $F$, i.e., the solutions of $F\left(\hat{x}^{*}\right)=\hat{x}^{*}$, are given as $x_{1}^{*}=x_{3}^{*}, x_{2}^{*}=$ $f\left(x_{1}^{*}\right), x_{3}^{*}=f\left(x_{2}^{*}\right)=f^{2}\left(x_{1}^{*}\right)$. Hence for any UCPO $\Sigma_{2}=\left\{x_{0}^{*}, x_{1}^{*}\right\}$ of (1), there corresponds a fixed point $\hat{x}^{*}=\left(x_{0}^{*} x_{1}^{*} x_{0}^{*}\right)^{T}$ of $F$, and vice versa. Hence the asymptotic stability of $\Sigma_{2}$ for (1) and (4) is equivalent to the asymptotic stability of the corresponding fixed point of $F$ for the system $\hat{x}(k+1)=F(\hat{x}(k))$. To analyze the latter, let us define the Jacobian of $F$ at equilibrium as $J=\left.\frac{\partial F}{\partial x}\right|_{\Sigma_{2}}$. The entries of $J$ can be calculated as $J(i, j)=\left.\frac{\partial Y_{i}}{\partial x_{j}}\right|_{\Sigma_{2}}, i, j=1,2,3$. After straightforward calculations, we obtain $J(1,1)=$
$J(1,2)=0, J(1,3)=1, J(2,1)=-K, J(2,2)=0$, $J(2,3)=a_{1}+K, J(3,1)=-K\left(a_{2}+K\right), J(3,2)=$ $-K, J(3,3)=\left(a_{1}+K\right)\left(a_{2}+K\right)$. The characteristic polynomial $p_{2}(\lambda)$ of $J$ can be calculated as:

$$
\begin{aligned}
p_{2}(\lambda)= & \operatorname{det}(\lambda I-J)=\lambda^{3}-\left(a_{1}+K\right)\left(a_{2}+K\right) \lambda^{2} \\
& +K\left(\left(a_{1}+K\right)+\left(a_{2}+K\right)\right) \lambda-K^{2} .
\end{aligned}
$$

Hence for the stability of $\Sigma_{2}$ for (1) and (4), we can study the Schur stability of $p_{2}(\lambda)$ given above. We will consider the Schur stability of $p_{2}(\lambda)$ for some cases in the sequel.

Now let us proceed to the general case $T=m$. Assume that (1) has an $m$ periodic UCPO given by $\Sigma_{m}=$ $\left\{x_{0}^{*}, x_{1}^{*}, \ldots, x_{m-1}^{*}\right\}$ and define $a_{1}=D f\left(x_{0}^{*}\right), a_{2}=$ $D f\left(x_{1}^{*}\right), \ldots, a_{m}=D f\left(x_{m-1}^{*}\right)$. In this case, by defining $x_{1}(k)=x(k-m), x_{2}(k)=x(k-m+1), \ldots, x_{m}(k)$ $=x(k-1), x_{m+1}(k)=x(k), \hat{x}=\left(x_{1} x_{2} \cdots x_{m+1}\right)^{T} \in$ $\mathbf{R}^{m+1}$, and $Y_{2}=f\left(x_{m+1}\right)+K\left(x_{m+1}-x_{1}\right)$, we can transform (1), (4) into the form $\hat{x}(k+1)=G(\hat{x}(k))$ where $G: \mathbf{R}^{m+1} \rightarrow \mathbf{R}^{m+1}$ is defined as $G(\hat{x})=$ $\left(x_{2} x_{3} \cdots x_{m+1} Y_{2}\right)^{T}$. As before, the UCPO $\Sigma_{m}$ does not correspond to a fixed point of $G$, but it corresponds to a fixed point of $F=G^{m}$. To see this, note that $F(\hat{x})=\left(Y_{1} Y_{2} \cdots Y_{m+1}\right)^{T}$ where $Y_{1}=x_{m+1}$, $Y_{i+1}=f\left(Y_{i}\right)+K\left(Y_{i}-x_{i}\right), i=1,2, \ldots, m$. For $\hat{x}^{*}=$ $\left(x_{1}^{*} x_{2}^{*} \cdots x_{m+1}^{*}\right)^{T}$, the fixed points of $F$, i.e., the solutions of $F\left(\hat{x}^{*}\right)=\hat{x}^{*}$, are given as $x_{i}^{*}=Y_{i}^{*}, i=$ $1, \ldots, m+1$, which in turn implies $x_{1}^{*}=x_{m+1}^{*}, x_{2}^{*}=$ $f\left(x_{1}^{*}\right), x_{j+1}^{*}=f\left(x_{j}^{*}\right), j=1, \ldots, m$. Hence the asymptotic stability of $\Sigma_{m}$ for (1) and (4) is equivalent to the asymptotic stability of the corresponding fixed point of $F$ for the system $\hat{x}(k+1)=F(\hat{x}(k))$. To analyze the latter, let us define the Jacobian of $F$ at the equilibrium as $J=\left.\frac{\partial F}{\partial x}\right|_{\Sigma_{2}}$. The entries of $J$ can be calculated as $J(i, j)=\left.\frac{\partial Y_{i}}{\partial x_{j}}\right|_{\Sigma_{m}}, i, j=1, \ldots, m+1$. After straightforward calculations, the entries of $J$ are found as follows: for $i=1, \ldots, m+1, j=1, \ldots, m$ we have

$J(i, j)= \begin{cases}0, & i-j<1, \\ -K, & i-j=1, \\ -K \prod_{l=j+1}^{i-1}\left(a_{l}+K\right), & i-j>1 .\end{cases}$

For $j=m+1$, we have

$J(1, m+1)=1$,

$J(i, m+1)=\prod_{l=1}^{i-1}\left(a_{l}+K\right), \quad i=2, \ldots, m+1$. 
Clearly the characteristic polynomial $p_{m}(\lambda)$ of $J$ has the following form:

$p_{m}(\lambda)=\lambda^{m+1}+c_{m} \lambda^{m}+\cdots+c_{1} \lambda+c_{0}$.

By using standard determinant formulas, after lengthy but straightforward calculations, the coefficients in (11) can be found as follows: (for $1<l<m$ )

$$
\begin{gathered}
c_{0}=-(-1)^{m} K^{m}, \quad c_{m}=-\prod_{i=1}^{m}\left(a_{i}+K\right), \\
c_{m-l}=-(-1)^{l} K^{l} \sum_{i_{1}=1}^{m} \sum_{i_{2}=i_{1}+1}^{m} \ldots \sum_{i_{l}=i_{l-1}+1}^{m} \\
\times \prod_{\substack{i=1 \\
i \neq i_{1}, \ldots, i_{l}}}^{m}\left(a_{i}+K\right) .
\end{gathered}
$$

Note that for $m=1$ and $m=2, p_{m}(\lambda)$ given by (11)(13) reduces to (6) and (8), respectively.

Now we can state our main results as follows. Let an $m$ period UCPO of (1) be given by $\Sigma_{m}=$ $\left\{x_{0}^{*}, x_{1}^{*}, \ldots, x_{m-1}^{*}\right\}$ and define $a_{1}=D f\left(x_{0}^{*}\right), a_{2}=$ $D f\left(x_{1}^{*}\right), \ldots, a_{m}=D f\left(x_{m-1}^{*}\right)$. Then:

(1) $\Sigma_{m}$ is exponentially stable for (1) and (4) if and only if $p_{m}(\lambda)$ given by (11)-(13) is Schur stable. This condition is only sufficient for asymptotic stability of $\Sigma_{m}$.

(2) If $p_{m}(\lambda)$ has at least one unstable root, i.e., magnitude strictly greater than unity, then $\Sigma_{m}$ cannot be stabilized by (1) and (4). Hence the proposed method to test stability is not conclusive only if some roots of $p_{m}(\lambda)$ are on the unit disc, i.e., have unit magnitude, while the rest of the roots are strictly inside the unit disc.

Remark 2. We note that the Schur stability of a polynomial can be checked by applying the so-called Schur-Cohn criterion, or equivalently the Jury test to the polynomial, see [23]. This test gives some necessary and sufficient conditions on the coefficients of the polynomial. These conditions are in the form of a finite set of inequalities, hence could be checked easily. In our case, once the terms $a_{i}$ are known, these conditions become some inequalities in terms of some polynomials of $K$. By finding the roots of these polynomials, we could determine the intervals of $K$ for which Schur stability holds. We will show some examples in the sequel.
At this point, note that one necessary condition for Schur stability of $p_{m}(\lambda)$ for any $m$ is that $p_{m}(1)>0$, see [23, p. 181]. This results in $p_{m}(1)=1+c_{m}+\cdots+$ $c_{1}+c_{0}>0$. By using (12), (12), this condition reduces to the following

$1-\prod_{i=1}^{m} a_{i}>0$.

This condition gives an inherent limitation of DFC in the sense that when it fails (in the sense that when $>$ sign is replaced by $<$ ), DFC cannot stabilize the corresponding $\Sigma_{m}$. We note that similar limitations in terms of some Floquet multipliers have been given in the literature, see $[18,21,24,25]$.

\section{Simulation results}

Now we will consider some special cases. For $m=1, p_{1}(\lambda)$ given by (6) is Schur stable if and only if (i) $1-a_{1}>0$ (see (14)), (ii) $1+a_{1}+2 K>0$, (iii) $K<1$, see [23, p. 180-183]. Clearly these inequalities are satisfied if and only if $-3<a_{1}<1$, see [18]. If this is the case, any $K$ satisfying $-\left(1+a_{1}\right) / 2<$ $K<1$ will result in the exponential stabilization of the corresponding UCPO. When $K>1$ or $K<$ $-\left(1+a_{1}\right) / 2$, at least one root of $p_{1}(\lambda)$ is unstable, hence the corresponding UCPO cannot be stable. For $K=1$ or $K=-\left(1+a_{1}\right) / 2$, stability cannot be deduced by using our approach.

To elaborate further, let us consider the logistic equation $f(x)=\mu x(1-x)$. For $\mu=3.7$, this map has one truly period 2 UCPO $\Sigma_{2}=\left\{x_{0}^{*}, x_{1}^{*}\right\}$ given by $x_{0}^{*}=0.390022, x_{1}^{*}=0.880248$. The fixed points $x_{A}=0, x_{B}=1-1 / \mu$ also induce period 2 orbits $\Sigma_{2 A}=\left\{x_{A}, x_{A}\right\}$ and $\Sigma_{2 B}=\left\{x_{B}, x_{B}\right\}$. However, one can easily show that the necessary condition (14) fails for these orbits, and hence they cannot be stabilized by DFC. For $\Sigma_{2}$, note that $a_{1}=\mu-2 \mu x_{0}^{*}=0.8138$, $a_{2}=\mu-2 \mu x_{1}^{*}=-2.8138$. The coefficients of $p_{2}(\lambda)$ are given by (8) as

$$
\begin{aligned}
& c_{2}=-\left(a_{1}+K\right)\left(a_{2}+K\right), \\
& c_{1}=K\left(\left(a_{1}+K\right)+\left(a_{2}+K\right)\right), \quad c_{0}=-K^{2} .
\end{aligned}
$$



if

From the Jury test, $p_{2}(\lambda)$ is Schur stable if and only

(i) $\left|c_{0}+c_{2}\right|<1+c_{1}$,

(ii) $\left|c_{1}-c_{0} c_{2}\right|<1-c_{0}^{2}$,

see [23, p. 180-183]. These inequalities are equivalent to the following:

(i) $1+2.29>0$,

(ii) $4 K^{2}-4 K-1.29>0$,

(iii) $2 K^{4}-2 K^{3}-4.29 K^{2}+2 K-1<0$,

(iv) $2 K^{3}+4.29 K^{2}-2 K-1<0$.

Clearly the sign conditions given above can be converted into some bounds on $K$ once the roots of these polynomials are found. By finding these roots, we conclude that $\Sigma_{2}$ can be exponentially stabilized if and only if $-0.3167<K<-0.2566$. Note that the precision of these bounds are related to the precision in obtaining the related polynomials and their roots. We performed a numerical simulation for this case with two different values of $K$ within the given range. Since the stabilization is only local, the DFC will work when the actual orbit of (1) is sufficiently close to $\Sigma_{2}$. To evaluate the exact domain of attraction for $\Sigma_{2}$ is very difficult, but by extensive numerical simulations we find that when

$d(i)=\sqrt{\sum_{j=0}^{2} d\left(x(i-j), \Sigma_{2}\right)^{2}}<0.09$

apparently the orbit is in the domain of attraction (note that the system is actually has dimension 3, see (7)). By using this idea, we simulated (1) and (4) with the following choice of input:

$u(k)= \begin{cases}K(x(k)-x(k-2)), & d(i)<0.09, \\ 0, & d(i) \geqslant 0.09 .\end{cases}$

Clearly, since the solutions of the logistic equation are chaotic in the uncontrolled case, eventually the control law given above will be effective and the stabilization of $\Sigma_{2}$ will be achieved for any $x(0) \in(0,1)$.

In the first simulation, we choose $K=-0.257$, which is quite close to the upper bound of the range of $K$ given above. The results of this simulation (with $\mu=3.7, K=-0.257, x(0)=0.4)$ are shown in Fig. 1, where we plotted $u(k)$ and $d\left(x(k), \Sigma_{2}\right)$ vs. $k$ in Fig. 1(a) and (b), respectively. In the second simulation, we choose $K=-0.28$, which is quite close to the middle of the range of $K$ given above. The results of this simulation (with $\mu=3.7, K=-0.28$, $x(0)=0.4)$ are shown in Fig. 1, where we plotted $u(k)$ and $d\left(x(k), \Sigma_{2}\right)$ vs. $k$ in Fig. 1(c) and (d), respectively. As can be seen, in both cases the decay of solutions to $\Sigma_{2}$ is exponential, and that the required input $u$ is sufficiently small and decays to zero exponentially as well. Moreover, as is evident in the Fig. 1, the decay is rather slow when $K$ is close to its stability boundaries, and relatively fast when $K$ is sufficiently away from the stability boundaries.

To show the change of the stability range for $K$, we performed similar analysis for various values of $\mu$. A similar analysis shows that for $\mu=3.75$, the stabilization is possible when $-0.3102<K<$ -0.30039 , and for $\mu=3.76$, the stabilization is possible when $-0.3090<K<-0.3089$. Similar analysis reveals that the stabilization is not possible for $\mu \geqslant 3.77$. Hence we conclude that there exists a critical value $3.76 \leqslant \mu^{*}<3.77$ such that DFC can be used for the stabilization of period 2 orbits for $\mu \leqslant \mu^{*}$, and cannot be used for $\mu>\mu^{*}$.

To elaborate further consider the case $m=3$. Let the UCPO be given as $\Sigma_{3}=\left\{x_{0}^{*}, x_{1}^{*}, x_{2}^{*}\right\}$, and define $a_{i}=D f\left(x_{i-1}^{*}\right), i=1,2,3$. The characteristic polynomial $p_{3}(\lambda)$ given by (11) has the coefficients

$$
\begin{aligned}
c_{3}= & -\left(a_{1}+K\right)\left(a_{2}+K\right)\left(a_{3}+K\right), \\
c_{2}= & K\left(\left(a_{1}+K\right)\left(a_{2}+K\right)+\left(a_{1}+K\right)\left(a_{3}+K\right)\right. \\
& \left.\quad+\left(a_{2}+K\right)\left(a_{3}+K\right)\right), \\
& =-K^{2}\left(\left(a_{1}+K\right)+\left(a_{2}+K\right)+\left(a_{3}+K\right)\right), \\
c_{1}=-K^{3} . &
\end{aligned}
$$

According to the Jury test, $p_{3}(\lambda)$ is Schur stable if and only if

(i) $\left|c_{0}\right|<1$,

(ii) $\left|c_{1}+c_{3}\right|<1+c_{0}+c_{2}$,

(iii) $\left|c_{2}\left(1-c_{0}\right)+c_{0}\left(1-c_{0}^{2}\right)+c_{3}\left(c_{0} c_{3}-c_{1}\right)\right|$

$$
<c_{0} c_{2}\left(1-c_{0}\right)+\left(1-c_{0}^{2}\right)+c_{1}\left(c_{0} c_{3}-c_{1}\right),
$$

see [23, pp. 180-183]. As an example, consider the logistic map with $\mu=3.85$. In this case, the logistic map has two true period 3 orbits given by $\Sigma_{3+}=$ $\{0.1725,0.5497,0.9529\}$ and $\Sigma_{3-}=\{0.4783,0.9606$, 

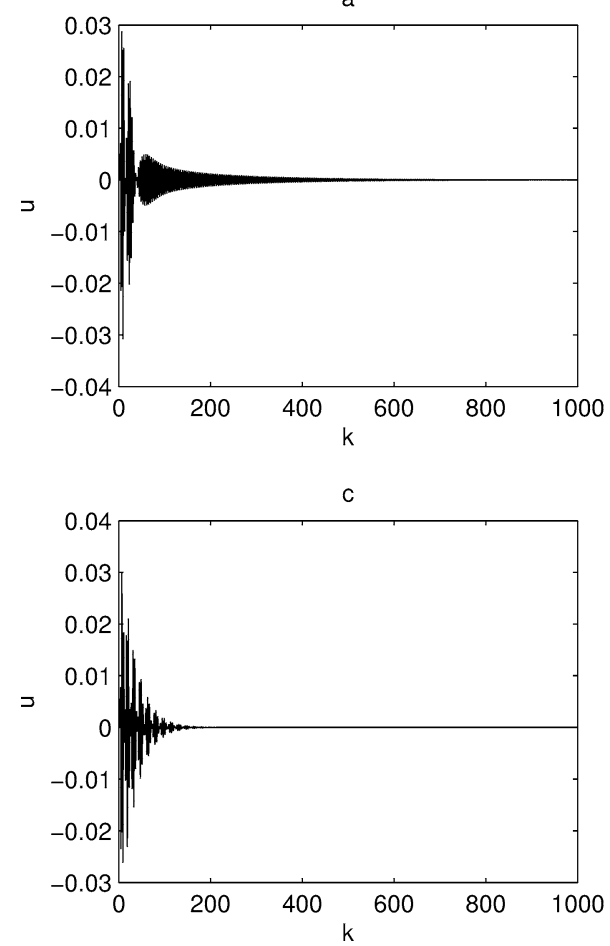
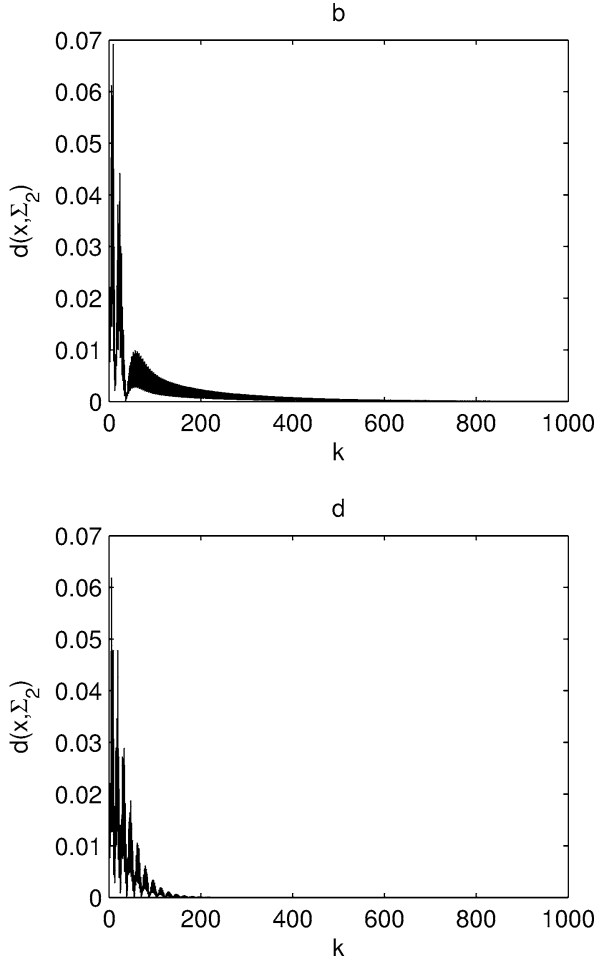

Fig. 1. The case $T=2, \mu=3.7, x(0)=0.4$. (a) $u(k)$ vs. $k$ for $K=-0.257$. (b) $d\left(x(k), \Sigma_{2}\right)$ vs. $k$ for $K=-0.257$. (c) $u(k)$ vs. $k$ for $K=-0.28$. (d) $d\left(x(k), \Sigma_{2}\right)$ vs. $k$ for $K=-0.28$.

$0.1453\}$. The fixed points $x_{A}=0$ and $x_{B}=1-$ $1 / \mu$ also induce period 3 orbits in the form $\Sigma_{3 A}=$ $\left\{x_{A}, x_{A}, x_{A}\right\}$ and $\Sigma_{3 B}=\left\{x_{B}, x_{B}, x_{B}\right\}$. One can easily show that the necessary condition (14) fails for $\Sigma_{3+}$ and $\Sigma_{3 A}$, and hence these orbits cannot be stabilized by DFC. For $\Sigma_{3 B}$, one can show that the Jury test, i.e., the inequalities (i)-(iii) given above, cannot be simultaneously satisfied for any $K$, hence DFC cannot be used for the stabilization $\Sigma_{3 B}$ as well. For $\Sigma_{3-}$, by evaluating these inequalities, one can show that DFC can be used for stabilization when $-0.1041<$ $K<-0.0315$. We performed a numerical simulation for this case with two different values of $K$ within the given range. Since the stabilization is only local, the DFC will work when the actual orbit of (1) is sufficiently close to $\Sigma_{3-}$. To evaluate the exact domain of attraction for $\Sigma_{3}$ - is very difficult, but by extensive numerical simulations we find that when

$$
d(i)=\sqrt{\sum_{j=0}^{3} d\left(x(i-j), \Sigma_{3-}\right)^{2}}<0.09
$$

apparently the orbit is in the domain of attraction (note that the system is actually has dimension 4 , see (7)). By using this idea, we simulated (1) and (4) with the following choice of input:

$u(k)= \begin{cases}K(x(k)-x(k-3)), & d(i)<0.09, \\ 0, & d(i) \geqslant 0.09 .\end{cases}$

Clearly, since the solutions of the logistic equation are chaotic in the uncontrolled case, eventually the control law given above will be effective and the stabilization of $\Sigma_{3}$ - will be achieved for any $x(0) \in(0,1)$.

In the first simulation, we choose $K=-0.032$, which is quite close to the upper bound of the range of $K$ given above. The results of this simulation (with $\mu=3.85, K=-0.032, x(0)=0.7$ ) are shown in Fig. 2, where we plotted $u(k)$ and $d\left(x(k), \Sigma_{3-}\right)$ vs. $k$ in Fig. 2(a) and (b), respectively. In this case, apparently the trajectories enter in the domain of attraction after $k=30$, and Fig. 2(b) is plotted for $k \geqslant$ 31 . In the second simulation, we choose $K=-0.06$, which is quite close to the middle of the range of $K$ 

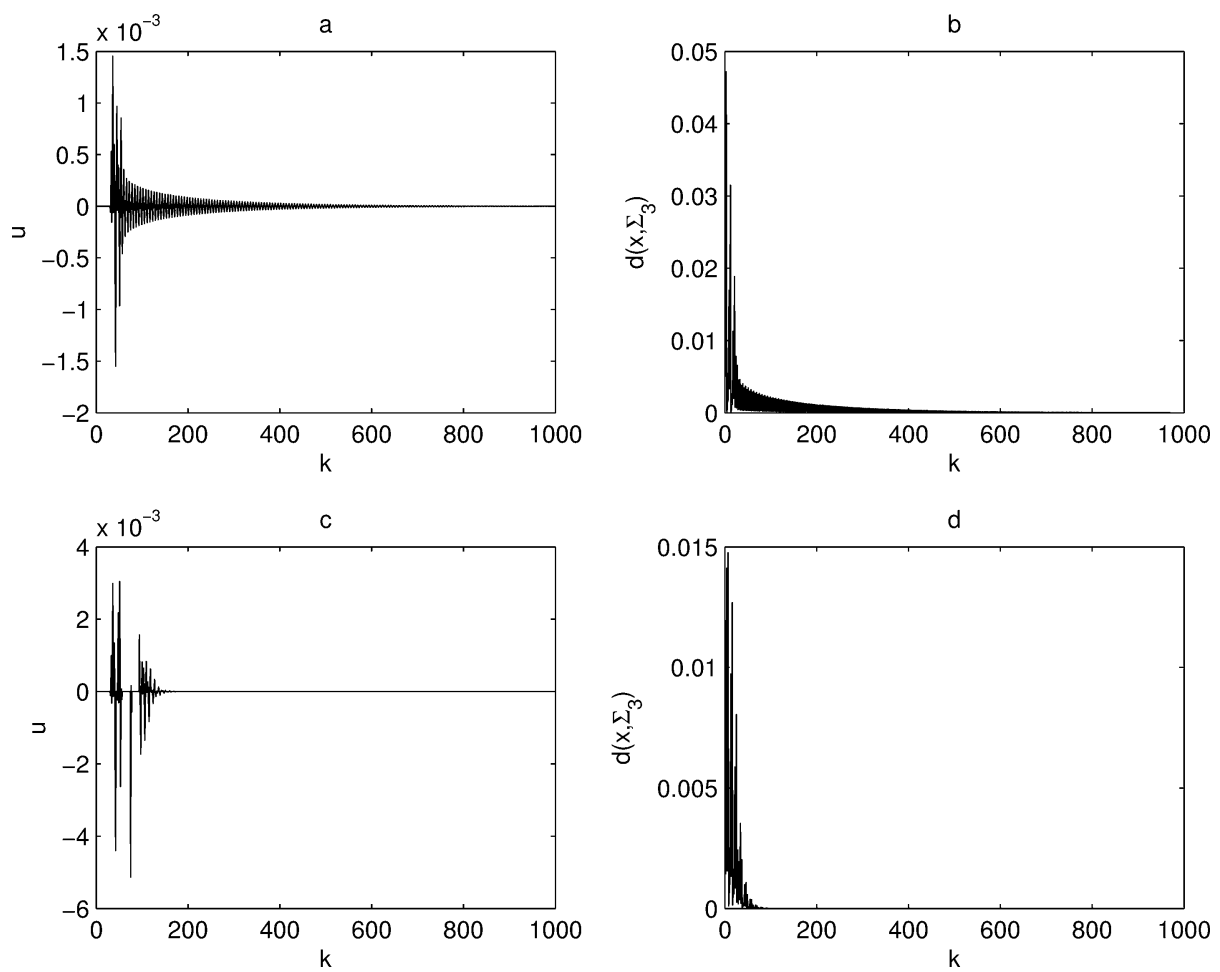

Fig. 2. The case $T=3, \mu=3.85, x(0)=0.7$. (a) $u(k)$ vs. $k$ for $K=-0.032$. (b) $d\left(x(k), \Sigma_{3-}\right)$ vs. $k$ for $K=-0.032$, plotted for $k \geqslant 31$. (c) $u(k)$ vs. $k$ for $K=-0.06$. (d) $d\left(x(k), \Sigma_{3-}\right)$ vs. $k$ for $K=-0.06$, plotted for $k \geqslant 91$.

given above. The results of this simulation (with $\mu=$ 3.85, $K=-0.06, x(0)=0.7)$ are shown in Fig. 2, where we plotted $u(k)$ and $d\left(x(k), \Sigma_{3-}\right)$ vs. $k$ in Fig. 2(c) and (d), respectively. In this case, apparently the trajectories enter in the domain of attraction after $k=91$, and Fig. 2(d) is plotted for $k \geqslant 91$. As can be seen, in both cases the decay of solutions to $\Sigma_{3-}$ is exponential, and that the required input $u$ is sufficiently small and decays to zero exponentially as well. Moreover, as is evident in the Fig. 2, the decay is rather slow when $K$ is close to its stability boundaries, and relatively fast when $K$ is sufficiently away from the stability boundaries.

To show the change of the stability range for $K$, we performed similar analysis for various values of $\mu$. A similar analysis shows that for $\mu=3.86$, the stabilization is possible when $-0.1024<K<-0.0615$, and for $\mu=3.87$, the stabilization is possible when $-0.1008<K<-0.087$. Similar analysis reveals that the stabilization is not possible for $\mu \geqslant 3.88$. Hence we conclude that there exists a critical value $3.87 \leqslant$ $\mu^{*}<3.88$ such that DFC can be used for the stabilization of period 3 orbits for $\mu \leqslant \mu^{*}$, and cannot be used for $\mu>\mu^{*}$.

\section{Conclusion}

In conclusion, we analyzed the stability of DFC for one-dimensional discrete time systems. We first constructed a map whose fixed points correspond to the periodic orbits of the uncontrolled chaotic system. Then the stability of DFC for the original chaotic system is equivalent to the stability of the corresponding fixed point of the constructed map. We derive the form of the characteristic polynomial of the Jacobian matrix of this map at the desired fixed point. Then the stability problem of DFC reduces to determine the Schur stability of the associated characteristic polynomial. By applying Jury test, we can determine the bounds on the gain of DFC to ensure the stability. 
The proposed methodology can also be applied to higher-dimensional discrete time chaotic systems, but this requires further research. Also extension of the results presented here to continuous time systems is not obvious, and this point deserves further investigation.

\section{References}

[1] E. Ott, C. Grebogi, J.A. Yorke, Phys. Rev. Lett. 64 (1990) 1196.

[2] T. Shinbrot, et al., Nature (London) 363 (1993) 411.

[3] G. Chen, X. Dong, From Chaos to Order: Methodologies, Perspectives and Applications, World Scientific, Singapore, 1999.

[4] K. Pyragas, Phys. Lett. A 170 (1992) 421.

[5] S. Bielawski, D. Derozier, P. Glorieux, Phys. Rev. E 49 (1994) R971.

[6] N.A. Loiko, A.V. Naumenko, S.I. Turovets, J. Exp. Theor. Phys. 85 (1997) 827.

[7] M.E. Brandt, H.T. Shih, G.R. Chen, Phys. Rev. E 56 (1997) R1334.

[8] K. Hall, et al., Phys. Rev. Lett. 78 (1997) 4518.
[9] F.J. Elmer, Phys. Rev. E 57 (1998) R4903.

[10] K. Pyragas, A. Tamaševičius, Phys. Lett. A 180 (1993) 99.

[11] D. Gauthier, et al., Phys. Rev. E 50 (1994) 2343.

[12] P. Parmananda, et al., Phys. Rev. E 59 (1999) 5266.

[13] J.E.S. Socolar, D.W. Sukow, D.J. Gauthier, Phys. Rev. E 50 (1994) 3245.

[14] K. Pyragas, Phys. Lett. A 206 (1995) 323.

[15] A. Kittel, J. Parisi, K. Pyragas, Phys. Lett. A 198 (1995) 433.

[16] K. Pyragas, Phys. Rev. Lett. 86 (2001) 2265.

[17] N. Baba, et al., Phys. Rev. Lett. 89 (2002) 4101.

[18] T. Ushio, IEEE Trans. Circuits Systems I Fund. Theory Appl. 43 (1996) 815.

[19] W. Just, et al., Phys. Rev. Lett. 78 (1997) 203.

[20] H. Nakajima, Phys. Lett. A 232 (1997) 207.

[21] H.G. Schuster, M.B. Stemmler, Phys. Rev. E 56 (1997) 6410.

[22] H.K. Khalil, Nonlinear Systems, 3rd Edition, Prentice-Hall, Upper Saddle River, 2002.

[23] S.N. Elaydi, An Introduction to Difference Equations, Springer-Verlag, New York, 1996.

[24] M. Giona, Nonlinearity 4 (1991) 911.

[25] T. Hino, S. Yamamoto, T. Ushio, Int. J. Bifur. Chaos Appl. Sci. Eng. 12 (2002) 439. 